\title{
Thermal effects in magnetoelectric memories with stress-mediated switching
}

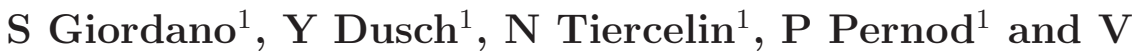 \\ Preobrazhensky ${ }^{1,2}$ \\ ${ }^{1}$ International Associated Laboratory LEMAC/LICS: \\ IEMN, UMR CNRS 8520, PRES Lille Nord de France, ECLille, 59651 Villeneuve \\ d'Ascq, France \\ ${ }^{2}$ Wave Research Center, Prokhorov General Physics Institute, Russian Academy of \\ Science, 38 Vavilov str., Moscow 119991, Russia \\ E-mail: Stefano.Giordano@iemn.univ-lille1.fr
}

\begin{abstract}
.
Heterostructures with magneto-electro-elastic coupling (e.g. multiferroics) are of paramount importance for developing new sensors, actuators and memories. With the progressive miniaturization of these systems it is necessary to take into account possible thermal effects, which may influence the normal operating regime. As paradigmatic example we consider a recently introduced non-volatile memory element composed of a magnetostrictive nanoparticle embedded in a piezoelectric matrix. The distribution of the physical fields in this matrix/inclusion configuration are determined by means of the Eshelby theory, the magnetization dynamics is studied through the Landau-LifshitzGilbert formalism, and the statistical mechanics is introduced with the Langevin and Fokker-Planck methodologies. As result of the combination of such techniques we determine the switching time between the states of the memory, the error probability and the energy dissipation of the writing process. They depend on the ratio $k_{B} T / v$ where $T$ is the absolute temperature and $v$ is the volume of the magnetoelastic particle.
\end{abstract}

PACS numbers: 85.70.-w, 85.50.-n, 75.80.+q, 05.10.Gg

Submitted to: J. Phys. D: Appl. Phys. 


\section{Introduction}

The possibility to attain a direct coupling between magnetic and electric properties in physical systems has recently stimulated a great scientific and technological interest $[1,2,3]$. At the beginning of these investigations different single-phase materials exhibiting the coexistence of ferroelectric and ferromagnetic responses were introduced (multiferroism) [4]. However, the development of these materials was strongly limited by the weak magneto-electric interaction at room temperature [5]. Therefore, to enhance and control the coupled response, composite structures of piezoelectric and magnetostrictive phases have been proposed and adopted in several devices [6, 7]. In this case the coupling is based on the principle of the mechanical stress (or strain) mediation.

Heterostructures based on intrinsic multiferroics or multi-phases materials are very promising from the energetic point of view (low-power devices). It is well known that the electric/mechanic reorientation of the magnetization in single particles or layers dissipates very low energies and it is appropriate for memories, spintronics and new logic paradigms $[8,9,10,11]$. The reduction of the energy dissipation is one of the most important factors for improving the integration level in memories and other devices [12]. However, when the energy of variables carrying the information is very low, thermal effects may play a crucial role and the so-called signal-to-noise ratio must be taken into account for studying the possible degradation of signals. For this reason typical temperature distributions in realistic structures have been recently investigated [13]. From the technological point of view the analysis of the compromise between operating temperature and miniaturization level is a central task for avoiding any form of information loss.

In this paper we investigate the possible thermal effects in a paradigmatic magnetoelectric memory element with stress-mediated switching. In particular, we consider a recently proposed heterostructure composed of a magnetoelastic particle embedded in a piezoelectric matrix $[14,15]$. We validated this approach by realising two macroscopic versions of this memory element $[16,17]$. Moreover, the assembly of the structure at the nanoscale is still in progress [18]. This device exhibits several technological advantages. In fact, it allows to avoid complex procedures for controlling the microstructure of interfaces in multiferroics, as recently proposed for enhancing the response of single-phase materials [19, 20, 21]. Moreover, although the direct control of magnetization by electric field was achieved at very low temperatures in some semiconductors [22, 23], our system is able to work at room temperature because of the stress-mediated coupling. Furthermore, the switching process, even if it consists in a very efficient non-toggle spin reorientation, it does not require electric pulses of particular duration and shape (e.g. for creating a temporary magnetic anisotropy). We remark that controlled pulses have been largely adopted in a variety of structures in the recent past $[24,25,26]$, and valuable simulations were performed to demonstrate their efficacy [27, 28]. 
The static and dynamic behavior of the memory element is described in Ref.[15] where, however, the temperature effects were neglected. Here, we perform the analysis of the dynamical response of this heterostructure at finite temperature and, in particular, we determine the switching time, the error probability and the energy dissipation associated to the process of writing a bit. These important quantities have been studied in terms of the ratio $k_{B} T / v$, describing the compromise between temperature and particle size. As an important result we develop a procedure for determining the maximum admissible value of the ratio $k_{B} T / v$. It means that we are able to find the maximum operating temperature if the size of the nanoparticle is fixed or, conversely, the minimum volume of the nanomagnet when the temperature is imposed.

The memory element here investigated is based on a couple of orthogonal states of the magnetization direction, generated by the competition between anisotropic and Zeeman energies [14]. The commutation process between the states is induced by the piezoelectric matrix, which is able to act on the particle through the magnetostrictive effect. The static behavior of the particle is described by a generalized energy function, able to allow for the spin-reorientation in terms of the applied electric field. Nanomechanical techniques [29, 30], based on the multi-physics Eshelby theory [31, 32], have been of primary importance for determining the distribution of the physical field within the heterogeneous structure. As for the dynamic response of the system, the above-mentioned analytical form of the energy function has been combined with classical ferromagnetic models [33, 34], in order to obtain the evolution equation of the magnetization direction during the switching phases. Moreover, in order to consider the temperature effects, the Landau-Lifshitz-Gilbert (LLG) equation [33, 34] has been generalized by means of the Brown formalism, i.e. by introducing a random field acting on the magnetization $[35,36,37,38]$. This approach leads to a stochastic Langevin equation or, equivalently, to a Fokker-Planck equation describing the time evolution of the density probability of the magnetization direction $[40,41]$. The numerical solution of the Langevin LLG stochastic equation allows us to obtain a complete picture on the dynamic behavior (i.e. the commutation strategy) of the system at finite temperature. In particular, these results are useful to design the device in agreement with the desired balance between operating temperature and particle size.

The structure of the paper is the following. In Section II we introduce the memory element and we describe the energy function governing its static response. In Sections III we briefly review the statistical mechanics of the magnetization in a single-domain particle. In particular, we discuss the Langevin and Fokker-Planck approaches, useful for the following developments. Finally, in Section IV we perform the complete thermal analysis of the memory element. We determine the switching time, the error probability and the energy dissipation associated to the commutation process in terms of the ratio $k_{B} T / v$. 


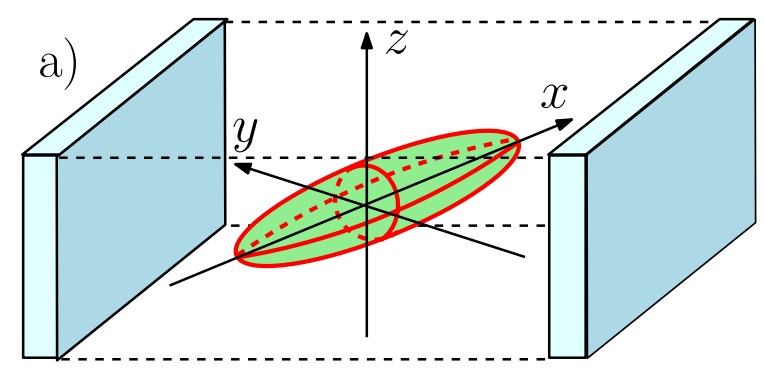

b)

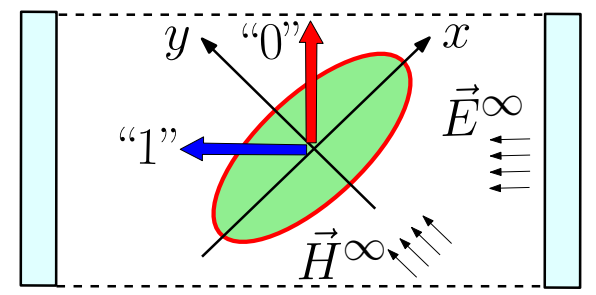

Figure 1. (Color online) Magnetoelastic particle inserted between two electrodes in a piezoelectric matrix: three-dimensional scheme (a) and top view (b). The easy axis and the hard axis of the particle are along the $x$ and $y$ axis, respectively. The electrodes generate the electric field $\vec{E}^{\infty}$ at $\varphi=3 \pi / 4$ while the magnetic field $\vec{H}^{\infty}$ is applied at $\varphi=\pi / 2$.

\section{The memory element}

We consider a magnetoelastic ellipsoidal particle embedded in a piezoelectric matrix (see Fig.1 for details) and we briefly introduce the formalism developed for modeling its behavior. The magnetic response of the particle is characterized by two different mechanism of anisotropy, namely the geometrical one generated by the prolate shape, and the physical one depending on the specific material and technological processes adopted. These anisotropies attempt to maintain the magnetization aligned along the $x$-axis (easy axis, EA). However, the principle of operation of the memory element is based on an externally applied magnetic field $\vec{H}^{\infty}$ aligned with the $y$-axis (hard axis, HA). Two stable states for the magnetization are in fact generated by the competition between the intrinsic anisotropies and the applied field (see state "0" and state "1" in Fig.1b). The piezoelectric matrix is used to change the state of the memory: the electric field $\vec{E}^{\infty}$ applied to the system generates a tension or a compression along the direction perpendicular to the planes of the parallel electrodes. More precisely, a tensile stress stores the state "1" while a compressive stress stores the state "0". This scheme corresponds to a non-toggle switching mechanism: it means that the knowledge of the previously stored state is not necessary for writing a new bit.

As a typical example, we adopt a nanoparticle made of $\mathrm{TbFe}_{2}$ (Terfenol) with semiaxes $a_{1}, a_{2}$, and $a_{3}$ inserted in a Lead Zirconate Titanate (PZT-5H) matrix [15]. The internal magnetization $\vec{M}=M_{s} \vec{\gamma}$ is uniform $\left(M_{s}\right.$ is its constant intensity and $\vec{\gamma}$ is a unit vector) because of the small size of the particle. The direction $\vec{\gamma}$ can be determined 
Table 1. Main parameters of the memory structure.

\begin{tabular}{llr}
\hline Parameter & Symbol & Value \\
\hline Axis along $x$ & $2 a_{1}$ & $45 \mathrm{~nm}$ \\
Axis along $y$ & $2 a_{2}$ & $25 \mathrm{~nm}$ \\
Axis along $z$ & $2 a_{3}$ & $20 \mathrm{~nm}$ \\
Magnetization & $M_{s}$ & $64 \times 10^{4} \mathrm{~A} / \mathrm{m}$ \\
Anisotropic effective field & $H_{a}$ & $18 \times 10^{4} \mathrm{~A} / \mathrm{m}$ \\
First magneto-elastic coefficient [43] & $\lambda_{111}$ & $1.7 \times 10^{-3}$ \\
Second magneto-elastic coefficient [43] & $\lambda_{100}$ & $0.1 \times 10^{-3}$ \\
Effective magneto-elastic coefficient [43] & $\lambda_{s}=\frac{3}{5} \lambda_{111}+\frac{2}{5} \lambda_{100}$ & $1.06 \times 10^{-3}$ \\
Young modulus of the particle & $E$ & $110 \mathrm{GPa}$ \\
Poisson ratio of the particle & $\nu$ & 0.35 \\
External magnetic field & $H^{\infty}$ & $50 \times 10^{4} \mathrm{~A} / \mathrm{m}$ \\
Distance between electrodes & $d$ & $130 \mathrm{~nm}$ \\
Applied voltage & $V$ & $\pm 0.5 \mathrm{~V}$ \\
Applied electric field & $E^{\infty}=-V / d$ & $\mu_{1}=\mu_{0}$ \\
Magnetic permeability & $A$ & $\pm 3.85 \times 10^{6} \mathrm{~V} / \mathrm{m}$ \\
Exchange stiffness constant (Terfenol) $[50]$ & $|\alpha|$ & $4 \pi \times 10^{-7} \mathrm{~A} / \mathrm{m}$ \\
Gilbert damping coefficient for RE-TM & $g$ & $10^{-11} \mathrm{~J} / \mathrm{m}$ \\
Landé g-factor & $\mu_{B}=\frac{e \hbar}{2 m_{e}}$ & 0.3 \\
Bohr magneton & $|\mathcal{G}|=\frac{g \mu_{B}}{\hbar}$ & 2 \\
Gyromagnetic ratio & & $1.76 \times 10^{11} \mathrm{rad} \mathrm{s} \mathrm{s}^{-1} \mathrm{~T}-1$ \\
\hline
\end{tabular}

by minimizing the following energy function [42]

$$
w(\vec{\gamma})=-\mu_{0} M_{s} \vec{\gamma} \cdot \vec{H}+\varphi_{a}(\vec{\gamma})-\hat{T}: \hat{\varepsilon}_{\mu}(\vec{\gamma})
$$

The first term (Zeemann energy) describes the effect of the local magnetic field $\vec{H}$. The second term $\varphi_{a}(\vec{\gamma})$ represents the anisotropic energy [14]. In our case we assume the usual uniaxial form $\varphi_{a}(\vec{\gamma})=-(1 / 2) \mu_{0} M_{s} H_{a} \gamma_{x}^{2}$. Finally, the third term represents the elastic energy, where $\hat{T}$ is the local stress and $\hat{\varepsilon}_{\mu}(\vec{\gamma})$ is the strain describing the magnetostriction. We use the standard expression $\hat{\varepsilon}_{\mu}(\vec{\gamma})=\left(\lambda_{s} / 2\right)(3 \vec{\gamma} \otimes \vec{\gamma}-\hat{I})$ where $\hat{I}$ is the identity tensor and the effective magnetostriction coefficient $\lambda_{s}$ can be evaluated as in Table 1 (where one can find the main parameters of the system) [43].

To conclude, we summarize the constitutive equations of the particle: the magnetic behavior is governed by $\vec{B}=\mu_{0}\left[\vec{H}+M_{s} \vec{\gamma}\right]$ where $\vec{B}$ is the magnetic induction and the elastic one by $\hat{T}=\hat{L}_{2}\left\{\hat{\varepsilon}_{0}-\hat{\varepsilon}_{\mu}(\vec{\gamma})\right\}$ where $\hat{\varepsilon}_{0}$ is the local strain tensor (referred to the demagnetized particle) and $\hat{L}_{2}$ is the stiffness tensor of the particle. The direction $\vec{\gamma}=\vec{\gamma}(\vec{H}, \hat{T})$ can be found through the minimization of Eq.(1). 

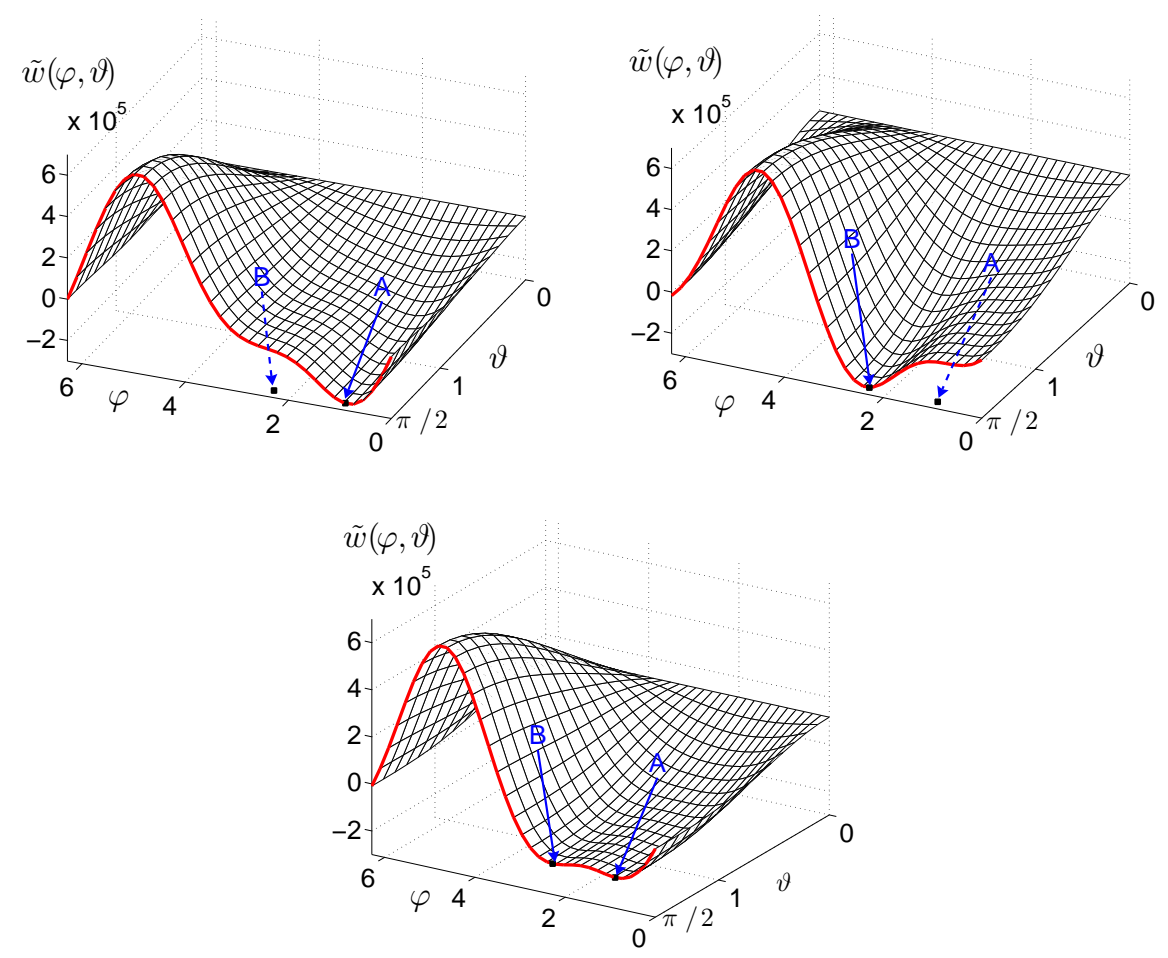

Figure 2. (Color online) Representation of $\tilde{w}(\vec{\gamma})\left[\mathrm{J} / \mathrm{m}^{3}\right]$ for different values of the applied voltage: $V=+0.5 \mathrm{~V}$ or $E^{\infty}=-3.85 \times 10^{6} \mathrm{~V} / \mathrm{m}$ (compression) in the first panel, $V=-0.5 \mathrm{~V}$ or $E^{\infty}=+3.85 \times 10^{6} \mathrm{~V} / \mathrm{m}$ (traction) in the second panel, and $V=0 \mathrm{~V}$ in third panel. Red curves correspond to $\vartheta=0$ and show the evolution of stable points $\mathrm{A}$ and $\mathrm{B}$.

\subsection{Coupling with the external magnetic field}

It is important to know the relationship between the local magnetic field $\vec{H}$ and the externally applied magnetic field $\vec{H}^{\infty}$. As recently discussed [15], the solution of this problem is given by

$$
\begin{aligned}
\vec{H} & =\left[\hat{I}-\hat{S}_{m}\left(\hat{I}-\hat{\mu}_{1}^{-1} \mu_{0}\right)\right]^{-1}\left[\vec{H}^{\infty}-\hat{S}_{m} \hat{\mu}_{1}^{-1} \mu_{0} M_{s} \vec{\gamma}\right] \\
& =\hat{A} \vec{H}^{\infty}+\hat{N} \vec{\gamma}
\end{aligned}
$$

where the tensor $\hat{S}_{m}$ is the magnetic Eshelby tensor [44, 45], $\mu_{0}$ is the vacuum magnetic permeability and $\hat{\mu}_{1}$ is the magnetic permeability tensor of the piezoelectric matrix. Tensors $\hat{A}$ and $\hat{N}$ can be directly identified by the first line of Eq.(2). The local magnetic field is therefore explicitly written in terms of the remotely applied magnetic field and of the internal magnetization orientation.

\subsection{Coupling with the external electric and elastic fields}

The coupling with the external electric and elastic fields is mediated by the piezoelectric matrix, representing the environment where the particle is inserted. We search for the 
relationship between the local stress $\hat{T}$ and the applied electric field $\vec{E}^{\infty}$ and the remote elastic strain $\hat{\varepsilon}^{\infty}$. We recall that the constitutive equation of the matrix can be written as $\hat{T}=\hat{L}_{1} \hat{\varepsilon}+\hat{Q}_{1} \vec{E}$ and $\vec{D}=\hat{R}_{1} \hat{\varepsilon}+\hat{\epsilon}_{1} \vec{E}$ where $\hat{L}_{1}$ is the elastic stiffness tensor, $\hat{\epsilon}_{1}$ is the permittivity tensor and $\hat{Q}_{1}$ and $\hat{R}_{1}=-\hat{Q}_{1}^{T}$ are the piezoelectric tensors of the matrix. The tensor properties of the PZT-5H matrix can be found in literature [46]. The magnetoelastic particle is inserted into the piezoelectric matrix with a specific initial magnetization direction $\vec{\gamma}_{0}$ and a corresponding magnetostriction $\hat{\varepsilon}_{\mu}\left(\vec{\gamma}_{0}\right)$. We define the local strain (within the particle) with respect to such an initial state and we therefore define $\hat{\varepsilon}=\hat{\varepsilon}_{0}-\hat{\varepsilon}_{\mu}\left(\vec{\gamma}_{0}\right)$. Here, $\hat{\varepsilon}_{0}$ is the local strain tensor referred to the demagnetized particle. Specifically, we observe that $\vec{\gamma}_{0}$ is aligned with the $x$-axis and therefore $\vec{\gamma}_{0}= \pm \vec{e}_{1}$ (where $\vec{e}_{i}$ is the unit vector along the $i$-th axis). Hence, the constitutive equations of the particle in the new reference frame read $\hat{T}=\hat{L}_{2}\left\{\hat{\varepsilon}-\left[\hat{\varepsilon}_{\mu}(\vec{\gamma})-\hat{\varepsilon}_{\mu}\left(\vec{\gamma}_{0}\right)\right]\right\}$ and $\vec{D}=\hat{\epsilon}_{2} \vec{E}$ where $\hat{L}_{2}$ and $\hat{\epsilon}_{2}$ are the elastic stiffness and the permittivity tensor of the particle, respectively.

The coupling problem can be approached and solved by means of the multi-physics Eshelby formalism [32, 31, 30, 15]. As recently verified [15], the local stress depends on the external electric and elastic fields and on the magnetization direction. In fact, we proved the explicit relation

$$
\hat{T}=\hat{C} \hat{\varepsilon}^{\infty}+\hat{D} \vec{E}^{\infty}+\hat{F}\left[\hat{\varepsilon}_{\mu}(\vec{\gamma})-\hat{\varepsilon}_{\mu}\left(\vec{\gamma}_{0}\right)\right]
$$

where the tensors $\hat{C}, \hat{D}$ and $\hat{F}$ can be calculated through the refined procedures described in literature [15]. They depend on the physical properties of the two phases and on the piezoelectric Eshelby tensor [47, 48, 49, 15].

\subsection{Static behavior of the system}

We can now combine previous results in order to obtain a generalized energy function describing the static behavior of the memory system. The set of equations describing the system is constituted of the energy minimisation, Eq.(1), the coupling with the external magnetic field $\vec{H}=\vec{H}\left(\vec{H}^{\infty}, \vec{\gamma}\right)$, Eq.(2), and the coupling with the external electric and elastic fields $\hat{T}=\hat{T}\left(\hat{\varepsilon}^{\infty}, \vec{E}^{\infty}, \vec{\gamma}\right)$, Eq.(3). This problem corresponds to the minimization of a new energy function defined as [15]

$$
\begin{aligned}
\tilde{w}= & -\mu_{0} M_{s} \vec{\gamma} \cdot \hat{A} \vec{H}^{\infty}-\frac{1}{2} \mu_{0} M_{s} \vec{\gamma} \cdot \hat{N} \vec{\gamma}+\varphi_{a}(\vec{\gamma}) \\
& -\hat{C} \hat{\varepsilon}^{\infty}: \hat{\varepsilon}_{\mu}(\vec{\gamma})-\hat{D} \vec{E}^{\infty}: \hat{\varepsilon}_{\mu}(\vec{\gamma}) \\
& -\frac{1}{2} \hat{F} \hat{\varepsilon}_{\mu}(\vec{\gamma}): \hat{\varepsilon}_{\mu}(\vec{\gamma})+\hat{F} \hat{\varepsilon}_{\mu}\left(\vec{\gamma}_{0}\right): \hat{\varepsilon}_{\mu}(\vec{\gamma}) .
\end{aligned}
$$

Such an expression provides the final magnetization orientation in terms of the external fields applied to the structure (the proof of Eq.(4) can be found in Appendix A). We can use the final form of the energy function to investigate the behavior of the memory element by letting $\hat{\varepsilon}^{\infty}=0, \vec{H}^{\infty}$ along the $y$-axis and $\vec{E}^{\infty}$ along the direction identified by $\varphi=\pi / 4, \vartheta=0$ (where $\varphi$ and $\vartheta$ are the standard spherical coordinates). When $E^{\infty}=0$ 
we observe two equivalent stable positions around $\varphi=\pi / 4, \vartheta=0$ and $\varphi=3 \pi / 4$, $\vartheta=0$ corresponding to the magnetization states represented in Fig.1. This is shown in the third panel of Fig.2 where $\tilde{w}$ is represented through a two-dimensional surface (in terms of $\varphi$ and $\vartheta$ ). The red curve corresponds to $\vartheta=0$ and shows two minima (points A and B). In the first panel of Fig.2 we have an applied compressive stress $\left(V=+0.5 \mathrm{~V}\right.$ and $\left.E^{\infty}=-3.85 \times 10^{6} \mathrm{~V} / \mathrm{m}\right)$ generating a single minimum point A state "0"). Conversely, in the second panel we have an applied tensile stress $(V=-0.5 \mathrm{~V}$ and $E^{\infty}=+3.85 \times 10^{6} \mathrm{~V} / \mathrm{m}$ ) corresponding to the minimum point B (state "1"). It is evident that the form of the energy function allows to obtain a non-toggle switching scheme for the memory element.

Some comments follow on the parameters adopted in our model. All the materialdependent parameters have been chosen in order to describe the Terfenol response for the magnetic particle and the PZT-5H response for the piezoelectric matrix. These materials represent classical paradigmatic examples being largely utilized in nanotechnology. The size of the system has been chosen at the nanoscale for assuring a mono-domain behavior of the magnetic particle (however, a varying volume of the particle has been considered in Section 4). The adopted anisotropic effective field $H_{a}$ is achievable with standard nanotechnologies $[14,15]$. A very important parameter is given by the external magnetic field $H^{\infty}$ : its value has been determined for fixing the stable states of the magnetization around $\varphi=\pi / 4$ and $\varphi=3 \pi / 4$. Therefore, the definition of this parameter is crucial for the correct operating of the memory element. Here we studied the position of the two minima of $\tilde{w}$ with a varying $H^{\infty}$ (and with $E^{\infty}=0$ ) and we selected the value corresponding to the geometry of Fig.1. We remark that $H^{\infty}$ depend on the material parameters above defined (i.e. on the physical properties of Terfenol and PZT-5H). To conclude, the applied electric field $E^{\infty}$ (or, equivalently the electric potential $V$ ) has been determined in order to have (i) a mechanical stress sufficient to observe the spin reorientation during the switching phases, and (ii) a switching time always in the subnanosecond scale. We obtained a value $V= \pm 0.5 \mathrm{~V}$, as largely discussed in Ref.[15]. This is a value highly compatible with most of microelectronic technologies.

While the dynamic analysis of the system has been performed in a recent paper [15], here we are interested in investigating the effect of the temperature. To do this we need to introduce the statistical mechanics of the magnetization.

\section{Statistical mechanics of magnetization in a single-domain particle}

The magnetic system is assumed to be monodomain and, therefore, all spins behave collectively. This is assured by the small size of the magnetic particle and by the high value of the exchange stiffness constant $A$ (see Table 1 for details) [50]. The dynamics of the magnetization direction $\vec{\gamma}$ is therefore described by the LLG equation [33, 34, 51]

$$
\frac{\mathrm{d} \vec{\gamma}}{\mathrm{d} t}=-\frac{\mathcal{G}}{M_{s}\left(1+\alpha^{2}\right)}\left[\vec{\gamma} \wedge \frac{\partial \tilde{w}}{\partial \vec{\gamma}}-\alpha \vec{\gamma} \wedge\left(\vec{\gamma} \wedge \frac{\partial \tilde{w}}{\partial \vec{\gamma}}\right)\right]
$$


where $\mathcal{G}$ is the gyromagnetic ratio, $\alpha$ is the Gilbert damping parameter and $\frac{\partial \tilde{w}}{\partial \vec{\gamma}}$ represents the effective field applied to the magnetic dipole (note that $\mathcal{G}<0$ and $\alpha<0$ for representing electrons precession). Here $\tilde{w}$ is the generalized energy function defined in Eq.(4). The previous LLG equation is valid for a system at $T=0^{\circ} \mathrm{K}$. In order to introduce thermal fluctuations we assume the Brown hypothesis affirming that the effects of the temperature can be mimicked by an addictive random field acting on the magnetization $[35,36,37,38]$. It means that we substitute $\frac{\partial \tilde{w}}{\partial \vec{\gamma}}$ with $\frac{\partial \tilde{w}}{\partial \vec{\gamma}}+\mathcal{D} \vec{n}$, where $\vec{n}$ is a stochastic process with three main properties: its average value is zero at any time, $\langle\vec{n}(t)\rangle=0$, it is completely uncorrelated (white), $\left\langle n_{i}(t) n_{j}(\tau)\right\rangle=2 \delta_{i j} \delta(t-\tau)$, and it is Gaussian. So, Eq.(5) is transformed into the Langevin LLG system

$$
\begin{aligned}
\dot{\varphi} \sin \vartheta= & -\frac{\mathcal{G}}{M_{s}\left(1+\alpha^{2}\right)} \\
& \times\left[\frac{\partial \tilde{w}}{\partial \vartheta}+\frac{\alpha}{\sin \vartheta} \frac{\partial \tilde{w}}{\partial \varphi}+\mathcal{D}(\vec{\delta}+\alpha \vec{\beta}) \cdot \vec{n}\right], \\
\dot{\vartheta}= & -\frac{\mathcal{G}}{M_{s}\left(1+\alpha^{2}\right)} \\
& \times\left[-\frac{1}{\sin \vartheta} \frac{\partial \tilde{w}}{\partial \varphi}+\alpha \frac{\partial \tilde{w}}{\partial \vartheta}+\mathcal{D}(\alpha \vec{\delta}-\vec{\beta}) \cdot \vec{n}\right],
\end{aligned}
$$

where we have introduced a mobile reference frame rigidly connected with the magnetization vector: $\vec{\delta}=(\cos \varphi \cos \vartheta, \sin \varphi \cos \vartheta,-\sin \vartheta), \vec{\beta}=(-\sin \varphi, \cos \varphi, 0)$ and $\vec{\gamma}=(\cos \varphi \sin \vartheta, \sin \varphi \sin \vartheta, \cos \vartheta)(\vartheta$ and $\varphi$ are the standard nutation and precession angles).

It is well known that the combination of dissipation (friction controlled by the Gilbert damping constant $\alpha$ ) and fluctuation (described by the diffusion coefficient $\mathcal{D}$ ) is able to describe the dynamic transient state leading to the equilibrium thermodynamics for long time [39, 40, 41]. It is a general concept valid both in classical mechanics $[52,53]$ and in quantum one $[54,55]$. The system obtained in Eq.(6) is a stochastic differential equation (SDE): from the mathematical point of view there are two different approaches for defining the meaning of a SDE, namely, the Itô stochastic calculus and the Stratonovich one $[56,57]$. Throughout all the paper we use the Stratonovich approach for two main reasons: firstly, the usual rules of calculus (for derivatives and integrals) remain unchanged and, secondly, the Stratonovich approach is the most convenient interpretation within the physical sciences since it can be obtained as the limiting process of a coloured noise towards an uncorrelated (white) one [40]. The typical tool for studying SDEs is the Fokker-Planck methodology based on a partial differential equation describing the dynamic of the density probability of the state of the system [40]. In our case the state of the system is given by the couple $(\varphi, \vartheta)$ and, therefore, the density probability can be written as $\rho=\rho(\varphi, \vartheta, t)$. The related Fokker-Planck equation assumes the form (see Appendix B for details)

$$
\frac{\partial \rho}{\partial t}=\frac{\mathcal{G}}{M_{s}\left(1+\alpha^{2}\right) \sin \vartheta} \frac{\partial}{\partial \varphi}\left\{\left[\frac{\partial \tilde{w}}{\partial \vartheta}+\frac{\alpha}{\sin \vartheta} \frac{\partial \tilde{w}}{\partial \varphi}\right] \rho\right\}
$$




$$
\begin{aligned}
& +\frac{\mathcal{G}}{M_{s}\left(1+\alpha^{2}\right)} \frac{\partial}{\partial \vartheta}\left\{\left[-\frac{1}{\sin \vartheta} \frac{\partial \tilde{w}}{\partial \varphi}+\alpha \frac{\partial \tilde{w}}{\partial \vartheta}\right] \rho\right\} \\
& -\frac{\mathcal{G}^{2} \mathcal{D}^{2}}{M_{s}^{2}\left(1+\alpha^{2}\right)} \frac{\partial}{\partial \vartheta}\left\{\frac{\cos \vartheta}{\sin \vartheta} \rho\right\} \\
& +\frac{\mathcal{G}^{2} \mathcal{D}^{2}}{M_{s}^{2}\left(1+\alpha^{2}\right)}\left\{\frac{1}{\sin ^{2} \theta} \frac{\partial^{2} \rho}{\partial \varphi^{2}}+\frac{\partial^{2} \rho}{\partial \vartheta^{2}}\right\} .
\end{aligned}
$$

As above said, this equation should have an asymptotic solution coherent with the equilibrium thermodynamics and, therefore, we can verify that

$$
\lim _{t \rightarrow \infty} \rho(\varphi, \vartheta, t)=\frac{\sin \vartheta}{\mathcal{Z}} \exp \left[-\frac{\tilde{w}(\varphi, \vartheta) v}{k_{B} T}\right]
$$

where the partition function $\mathcal{Z}$ is given by

$$
\mathcal{Z}=\int_{0}^{\pi} \int_{0}^{2 \pi} \sin \vartheta \exp \left[-\frac{\tilde{w}(\varphi, \vartheta) v}{k_{B} T}\right] d \varphi d \vartheta
$$

Here $k_{B}$ is the Boltzmann constant and $T$ is the absolute temperature. Note that the term $\sin \vartheta$ in previous expressions is due to the (non-cartesian) spherical system (it corresponds to the Jacobian of the coordinates transformation). Moreover, $v$ represents the volume of the magnetic particle ( $\tilde{w} v$ is the total energy being $\tilde{w}$ the energy density). The value of the diffusion constant $\mathcal{D}$ can be found by substituting Eq.(8) in Eq.(7) and by observing that we obtain an identity if and only if

$$
\mathcal{D}^{2}=\frac{\alpha M_{s} k_{B} T}{\mathcal{G} v}
$$

an equation representing the specific fluctuation-dissipation property.

The obtained Fokker-Planck equation is particularly useful for obtaining a simplified version of the Langevin LLG system: in fact, in Eq.(6) a three-dimensional random vector has been added for introducing the fluctuations in a system with two variables $(\varphi$ and $\vartheta$ ). There is no need to embed the system in a three-dimensional space and, moreover, there are important reasons for not doing so (coherence/elegance of the theory and saving of computational resources). We consider the following new version of the Langevin LLG system where only two noise terms are considered [58, 59, 60]

$$
\begin{aligned}
\dot{\varphi}= & -\frac{\mathcal{G}}{M_{s}\left(1+\alpha^{2}\right) \sin \vartheta}\left[\frac{\partial \tilde{w}}{\partial \vartheta}+\frac{\alpha}{\sin \vartheta} \frac{\partial \tilde{w}}{\partial \varphi}\right] \\
& +\frac{1}{\sin \vartheta} \sqrt{\frac{1}{2 \tau_{N}}} n_{\phi}, \\
\dot{\vartheta}= & -\frac{\mathcal{G}}{M_{s}\left(1+\alpha^{2}\right)}\left[-\frac{1}{\sin \vartheta} \frac{\partial \tilde{w}}{\partial \varphi}+\alpha \frac{\partial \tilde{w}}{\partial \vartheta}\right] \\
& +\frac{1}{2 \tau_{N}} \frac{\cos \vartheta}{\sin \vartheta}+\sqrt{\frac{1}{2 \tau_{N}}} n_{\theta} .
\end{aligned}
$$

Here we have introduced to so-called Néel time

$$
\tau_{N}=\frac{M_{s}\left(1+\alpha^{2}\right) v}{2 \alpha \mathcal{G} k_{B} T},
$$


representing the characteristic response time of a particle without external fields. If the noises have the standard properties $\left\langle n_{\phi}(t)\right\rangle=0,\left\langle n_{\theta}(t)\right\rangle=0,\left\langle n_{\phi}(t) n_{\theta}(\tau)\right\rangle=0$, $\left\langle n_{\phi}(t) n_{\phi}(\tau)\right\rangle=2 \delta(t-\tau),\left\langle n_{\theta}(t) n_{\theta}(\tau)\right\rangle=2 \delta(t-\tau)$ and they are Gaussian, we can prove that the Fokker-Planck equation established starting form Eq.(11) are exactly coincident to Eq.(7). From the theoretical point of view Eq.(11) is more coherent and elegant since the SDE lives completely on the spherical surface without the need for a three-dimensional embedding (it represents the covariant formulation of the SDE on the spherical manifold) [59, 60]. Moreover, from the computational point of view Eq.(11) is convenient since two random numbers must be generated at any time step, instead of the three ones needed for the implementation of Eq.(6). They can be directly obtained by means of the Box-Muller theorem [61]. Another remarkable advantage of Eq.(11) is that the noise induced drift term is always zero, yielding exactly the same SDE both for the Itô and the Stratonovich approach. This fact allows us to apply indifferently numerical techniques specifically developed for either the Itô or the Stratonovich interpretation of SDEs (see Appendix C for details).

To conclude, when we approach the problem of studying the thermal effects on a single particle we can adopt one of the three following methodologies. First, we can take into consideration the Fokker-Planck equation and we can search its solution through the finite difference method or the finite element method. Such a technique has been used to investigate the dependence of the magnetization reversal on temperature, damping and applied fields $[62,63,64]$. As second approach, it is possible to develop the density probability in a series of harmonic functions and to analyse the dynamics of related coefficients. The kinetic equation for these coefficients has been obtained [65, 66, 67] and it has been largely used for determining the relaxation time of the Fokker Planck operator $[68,69,70,71]$. Finally, the third approach consists in numerically solving the Langevin equation and in calculating the relevant average values through the Monte Carlo method [72]. In the following we adopt this approach with a standard integration scheme discussed in Appendix C.

\section{Switching process within the magnetoelectric memory}

We can now approach the problem of evaluating the temperature effects on the memory element. As one can find in Table 1, we have considered an ellipsoidal particle with axes of lengths 45, 25 and $20 \mathrm{~nm}$. Nevertheless, we may now consider an arbitrary size of the particle. To explain this point we recall an important property of the Eshelby theory (which is valid for the case with any possible coupling): when an ellipsoidal particle is embedded in an infinite matrix and subjected to uniform external actions, the physical fields (electric, magnetic and elastic) induced within the particle itself are always uniform and they depend on the material properties of the two phases and on the ratios $a_{1} / a_{2}$ and $a_{2} / a_{3}[32,31,30,15,47,48,49]$. The internal fields do not depend on the actual size of the particle: only the shape of the ellipsoid may influence the particle response. Therefore, also the results based on the energy function defined in Eq.(4) are 
scale invariant and depend only on the ratio between the axes lengths. We conclude that the numerical evaluation of $\tilde{w}$ described in Section II can be used for any rescaled version of the particle.

The only effect of the real size of the nanomagnet is introduced in the Langevin system (see Eq.(11)) through the Néel time $\tau_{N}$ defined in Eq.(12). Since $1 / \tau_{N}$ is directly proportional to $k_{B} T / v$ (with a coefficient that is simply material dependent) we can analyse the thermal effects in terms of the ratio $k_{B} T / v$, describing the conflict between temperature and particle size. We remark that one of the most important parameter of the system is the energy barrier between the states A and B, which can be observed in the third panel of Fig.2 (in absence of the electric field). It is an intrinsic property of the structure depending only on the anisotropies (geometrical and physical) of the particle and on the externally applied magnetic field (creating the quite orthogonal states): with the set of parameters defined in Table 1 we obtain an energy barrier equals to $\Delta e=2.5 \times 10^{4} \mathrm{~J} / \mathrm{m}^{3}$. It is evident that the memory can work only if the density of thermal energy $k_{B} T / v$ is much lower than $\Delta e$ (for avoiding unwanted switching between the states). For example, for the initially proposed structure $\left(v \simeq 10^{-22} \mathrm{~m}^{3}\right)$ at room temperature $\left(T=300^{\circ} \mathrm{K}\right)$ we have $k_{B} T / v=40 \mathrm{~J} / \mathrm{m}^{3} \ll \Delta e$ and, therefore, the system should work correctly. In this case the total energy barrier between the states $\mathrm{A}$ and B corresponds to $v \Delta e=1.7 \cdot 10^{-18} \mathrm{~J} \simeq 400 k_{B} T$. It is interesting to know how much we can increase the temperature or, on the other hand, decrease the volume of the particle, without modifying the regular operation of the device. In other words, we search for the maximum value of $k_{B} T / v$ admissible for our structure. In order to do this, we consider the system without electric field applied to the piezoelectric matrix and we suppose to have an initial magnetization in the state $\mathrm{A}$ or B. We observe that the state $\mathrm{A}$ is represented by $\vec{\gamma}=\vec{v}_{A}=\left(\cos \varphi_{A}, \sin \varphi_{A}, 0\right)$ where $\varphi_{A} \simeq 0.892$ while for the state $\mathrm{B}$ we have $\vec{\gamma}=\vec{v}_{B}=\left(\cos \varphi_{B}, \sin \varphi_{B}, 0\right)$ where $\varphi_{B} \simeq 2.277$. We determine the trajectories of $\vec{\gamma}$ starting from these points in order to analysing the stability of the stored bit. It means that we simulate the transition $\mathrm{A} \rightarrow \mathrm{A}$ generated by the electric potential change $V=+0.5 \mathrm{~V} \rightarrow V=0 \mathrm{~V}$ and the transition $\mathrm{B} \rightarrow \mathrm{B}$ generated by the electric potential change $V=-0.5 \mathrm{~V} \rightarrow V=0 \mathrm{~V}$. For any value of the ratio $k_{B} T / v(100$ equispaced values in the range from $10 \mathrm{~J} / \mathrm{m}^{3}$ to $2 \times 10^{4} \mathrm{~J} / \mathrm{m}^{3}$ ) we generate a large number (dynamically adjusted from $10^{4}$ for the high values of $k_{B} T / v$ to $5 \times 10^{6}$ for the low values of $k_{B} T / v$ ) of trajectories $\vec{\gamma}(t)$ by solving Eq.(11) (with $E^{\infty}=0$ and $\vec{\gamma}(0)=\vec{v}_{A}$ or $\vec{\gamma}(0)=\vec{v}_{B}$ ) and we determine the average values through the Monte Carlo method. The numerical solution of Eq.(11) has been performed through the integration scheme discussed in Appendix $\mathrm{C}$ with a time step $\delta t=2.4 \times 10^{-13}$ sec.

The results are reported in Figs.3 and 4. In the first panel we show the time evolution of the three components of $\langle\vec{\gamma}(t)\rangle$ for the extreme values of the ratio $k_{B} T / v$. It is evident that for the larger values of $k_{B} T / v$ (dashed lines in the first panel of Figs.3 and 4) we observe a vector $\langle\vec{\gamma}\rangle$ rapidly aligned to the $y$-axis, indicating the complete information loss (in this situation we have $\vec{\gamma} \cdot \vec{v}_{A} \simeq \vec{\gamma} \cdot \vec{v}_{B}$ ). A measure of this effect is given by the error probability, shown in the second panel of Figs.3 and 

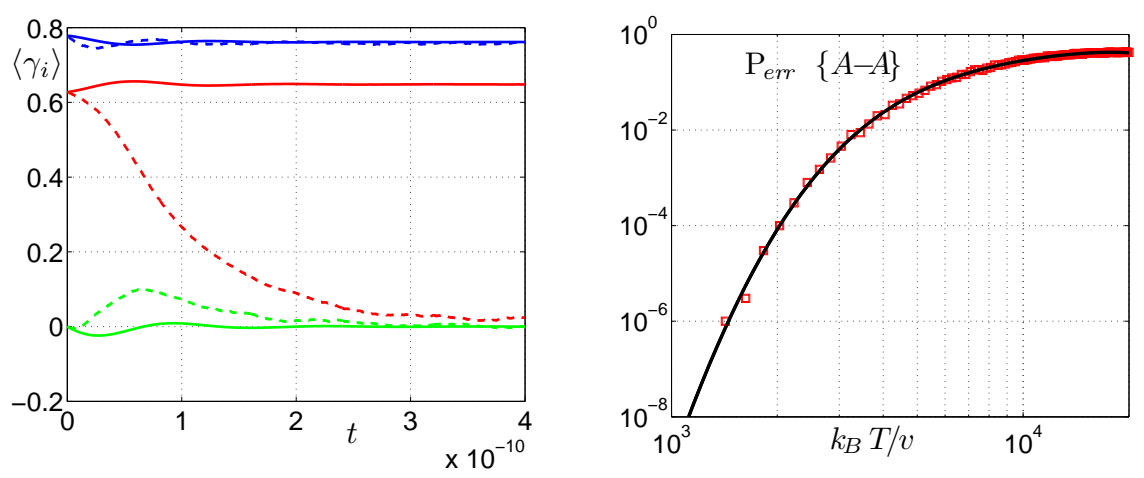

Figure 3. (Color online) Stability analysis of the switching A $(V=+0.5 \mathrm{~V})$ - A $(V=0 \mathrm{~V})$. First panel: trajectories followed by the average magnetization components (red: $\left\langle\gamma_{x}\right\rangle$; blue: $\left\langle\gamma_{y}\right\rangle$; green: $\left\langle\gamma_{z}\right\rangle$ ). Solid lines correspond to the smallest ratio $k_{B} T / v$ $\left(10 \mathrm{~J} / \mathrm{m}^{3}\right)$ while dashed lines to the highest one $\left(20000 \mathrm{~J} / \mathrm{m}^{3}\right)$. Second panel: error probability in terms of the ratio $k_{B} T / v$.
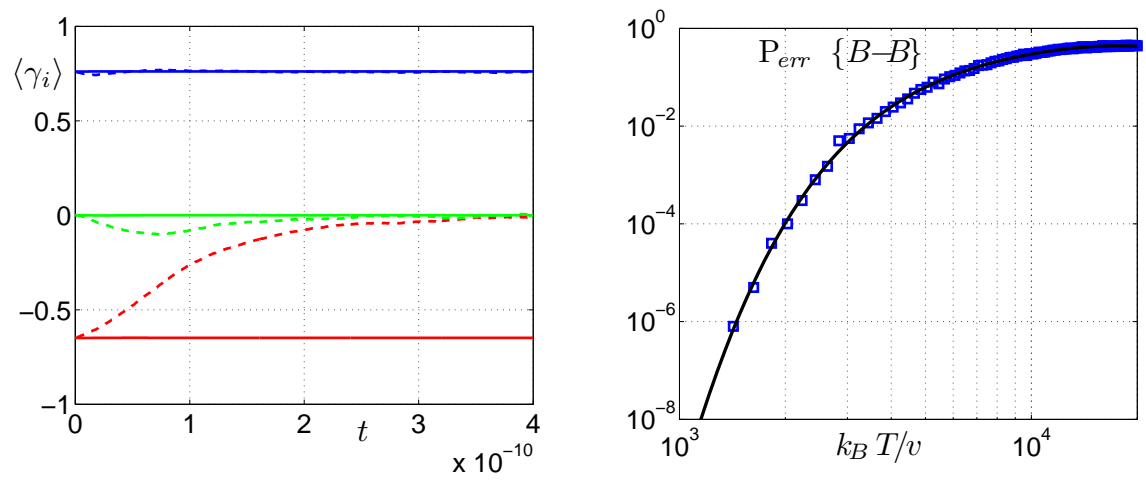

Figure 4. (Color online) Stability analysis of the switching $\mathrm{B}(V=-0.5 \mathrm{~V})$ - B $(V=0 \mathrm{~V})$. The panels description can be found in Fig.3.

4. For any value of $k_{B} T / v$ we follow $N_{t}$ trajectories for a long time and we determine the number of unwanted switching towards the other state. The error probability is given by the ratio between this number and the total number $N_{t}$ of trajectories. Such a number $N_{t}$ is dynamically adjusted and it corresponds to $10^{4}$ for the higher values of $k_{B} T / v$ (sizeable error probability) and to $5 \times 10^{6}$ for the lower values of $k_{B} T / v$ (very low error probability). We remark that it is difficult to have a good estimate of the error probability for values of the ratio $k_{B} T / v$ much lower than $10^{3} \mathrm{~J} / \mathrm{m}^{3}$ since this probability is very low and we should use a large number of trajectories $N_{t} \gg 10^{8}$, which is quite prohibitive from the computational point of view. We obtained quite the same curve of $P_{\text {err }}$ versus $k_{B} T / v$ for both transitions A-A and B-B. It means that the error probability is a symmetric quantity for our system. Moreover, we observe that if $k_{B} T / v \rightarrow+\infty$ then $P_{\text {err }} \rightarrow 1 / 2$, a value exactly quantifying the total information loss. We can identify the maximum value admissible for $k_{B} T / v$ in order to have a negligible error probability: for example, if we impose $P_{e r r} \ll 10^{-8}$ then we obtain 

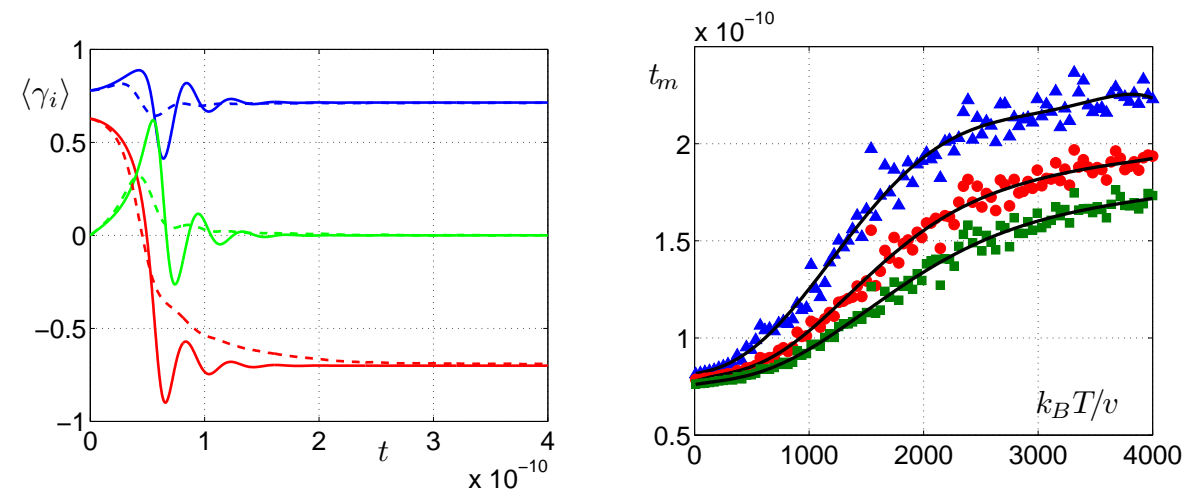

Figure 5. (Color online) Switching process between states A and B (traction, $V=$ $-0.5 \mathrm{~V})$. First panel: trajectories followed by the average magnetization components (red: $\left\langle\gamma_{x}\right\rangle$; blue: $\left\langle\gamma_{y}\right\rangle$; green: $\left\langle\gamma_{z}\right\rangle$ ). Solid lines correspond to the smallest ratio $k_{B} T / v\left(10 \mathrm{~J} / \mathrm{m}^{3}\right)$ while dashed lines to the highest one $\left(4 \times 10^{3} \mathrm{~J} / \mathrm{m}^{3}\right)$. Second panel: switching time $t_{m}$ versus $k_{B} T / v$ for three different values of the precision parameter (blue triangles: $\epsilon_{\gamma}=0.01$; red circles: $\epsilon_{\gamma}=0.02$; green squares: $\epsilon_{\gamma}=0.03$ ).
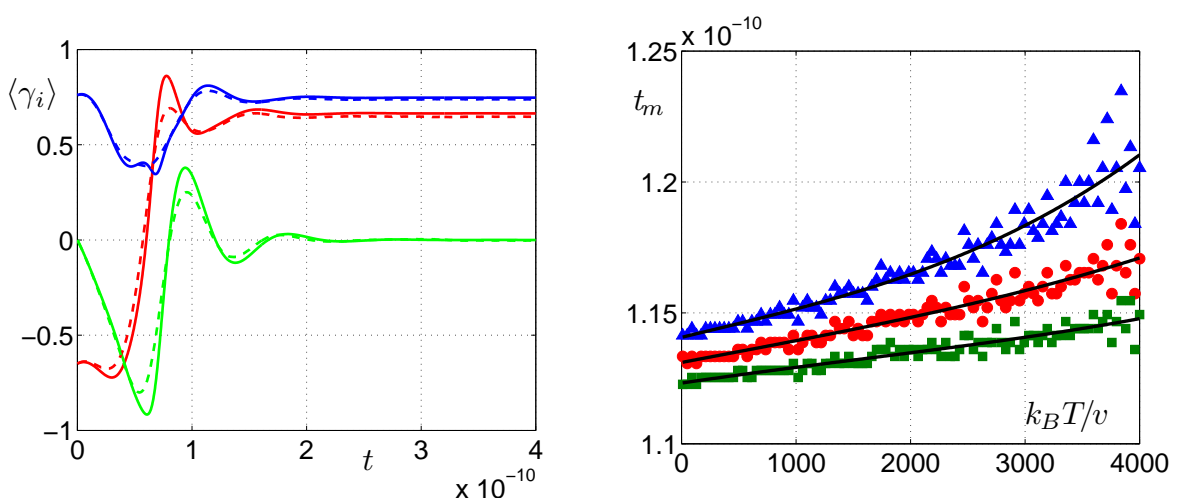

Figure 6. (Color online) Switching process between states B and A (compression, $V=+0.5 \mathrm{~V})$. The panels description can be found in Fig.5.

$\left(k_{B} T / v\right)_{\max } \simeq 10^{3} \mathrm{~J} / \mathrm{m}^{3}$ (see second panel of Figs.3 and 4), which is much larger than our initial proposition $\left(40 \mathrm{~J} / \mathrm{m}^{3}\right)$ and, at the same time, much smaller than the fixed energy barrier between the states $\left(\Delta e=2.5 \times 10^{4} \mathrm{~J} / \mathrm{m}^{3}\right)$. Of course one can select an arbitrary threshold for the error probability by obtaining a different maximum value admissible for $k_{B} T / v$. This stability analysis was performed by starting from a deterministic initial condition (given by $\vec{\gamma}(0)=\vec{v}_{A}$ or $\vec{\gamma}(0)=\vec{v}_{B}$ ) since we were interested in examining the possible escape from the potential wells induced by the thermal agitation during the time evolution. It is the possibility to remain inside the wells that is important to evaluate the pertinent error probability.

Once determined the restriction on the ratio $k_{B} T / v$ we may analyse the dynamics of the transitions $\mathrm{A}-\mathrm{B}$ and $\mathrm{B}-\mathrm{A}$. In this case, since we want to analyse the real dynamics at a given temperature, we can not start our simulations at $\vec{\gamma}(0)=\vec{v}_{A}$ or $\vec{\gamma}(0)=\vec{v}_{B}$. Instead, we must start with a random initial condition coherent with the statistical 


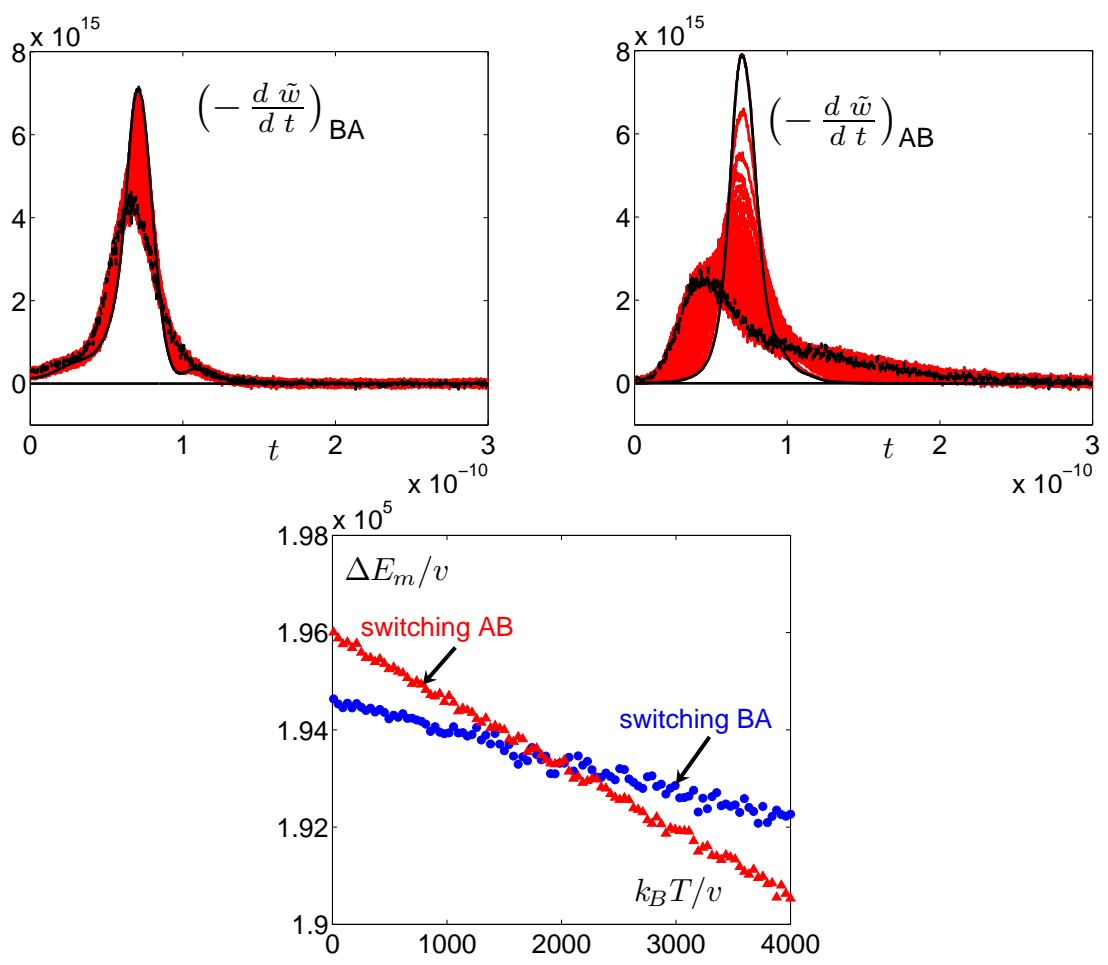

Figure 7. (Color online) Energy dissipation during switching phases. First panel: average value of $-d \tilde{w} / d t$ during the transition B-A for different values of $k_{B} T / v(100$ equispaced values from $10 \mathrm{~J} / \mathrm{m}^{3}$ to $4000 \mathrm{~J} / \mathrm{m}^{3}$ ). The black continuous line corresponds to the smallest ratio $k_{B} T / v$ while the black noisy line to the highest one. Second panel: average value of $-d \tilde{w} / d t$ during the transition A-B. The black lines have the same meaning as in the first panel. Third panel: magnetic dissipated energy $\Delta E_{m} / v$ in terms of $k_{B} T / v$ for both transitions A-B and B-A.

distribution within the initial potential well. The initial density probability corresponds to the thermodynamic equilibrium and it is therefore given by Eqs.(8) and (9). We extracted the initial conditions from this distribution. For any value of $k_{B} T / v$ in the range from $10 \mathrm{~J} / \mathrm{m}^{3}$ to $4 \times 10^{3} \mathrm{~J} / \mathrm{m}^{3}$ we generate $10^{4}$ trajectories of the magnetization and we evaluate their average values. We have chosen a range of $k_{B} T / v$ exceeding the threshold value $\left(k_{B} T / v\right)_{\max } \simeq 10^{3} \mathrm{~J} / \mathrm{m}^{3}$ for better exploring the critical region. In Fig. 5 we show the results for the switching A-B corresponding to the applied traction at $V=-0.5 \mathrm{~V}$. Similarly, in Fig. 6 we show the results for the switching B-A corresponding to the applied compression at $V=+0.5 \mathrm{~V}$. In the first panel we present the time behavior of the components of $\langle\vec{\gamma}\rangle$. The good switching behavior is evident in the whole range of variation of $k_{B} T / v$ : in the first panel of Figs.5 and 6 solid lines correspond to the smallest ratio $k_{B} T / v\left(10 \mathrm{~J} / \mathrm{m}^{3}\right)$ while dashed lines to the highest one $\left(4 \times 10^{3} \mathrm{~J} / \mathrm{m}^{3}\right)$. In particular, the regular accomplishment of transitions is well described by the $\operatorname{limit} \lim _{t \rightarrow \infty} \vec{\gamma} \cdot \vec{v}_{B}=1$ for the switching A-B and by $\lim _{t \rightarrow \infty} \vec{\gamma} \cdot \vec{v}_{A}=1$ for the switching B-A. We use these asymptotic behavior to introduce the switching time $t_{m}$ of the process. It is defined as the first instant of time $t_{m}$ satisfying the condition $\left|\vec{\gamma}(t) \cdot \vec{v}_{B}-\vec{\gamma}(+\infty) \cdot \vec{v}_{B}\right|<\epsilon_{\gamma}$ for any 
$t>t_{m}$ (for the transition A-B). Evidently, for the second transition B-A the inequality reads $\left|\vec{\gamma}(t) \cdot \vec{v}_{A}-\vec{\gamma}(+\infty) \cdot \vec{v}_{A}\right|<\epsilon_{\gamma}$. Note that the precision parameter $\epsilon_{\gamma}$ (around $10^{-2}$ ) concerns the accuracy of the measurement of the magnetization $\vec{\gamma}$ within the asymptotic regime and it is not related with the error probability above defined $\left(P_{\text {err }} \ll 10^{-8}\right.$ if $\left.k_{B} T / v<10^{3} \mathrm{~J} / \mathrm{m}^{3}\right)$. In the second panel of Figs.5 and 6 we show the switching time in terms of the ratio $k_{B} T / v$. The three different set of data correspond to three values of the precision parameter $\epsilon_{\gamma}$. In all cases we observe an increasing trend of $t_{m}$ versus $k_{B} T / v$. Of course, the switching times obtained in Figs.5 and 6 (hundreds of ps) must be realistically augmented in order to consider different phenomena not contemplated in our model: response time of the piezoelectric matrix, time to transfer the stress to the magnetic particle, and delays of the electronic system generating the electric pulses. We can estimate a loss of velocity of about ten times, resulting in final switching times of some nanoseconds. However, the values of the writing time are in any case strongly competitive with other standard or spintronic memory technologies [73]. The behavior of the curves for the switching A-B and B-A is quite different because of the different physical processes involved. As a matter of fact, the transition A-B is characterised by a traction inducing a planar anisotropy from the magnetic point of view, while the transition B-A is characterised by a compression inducing an axial anisotropy for the magnetization.

To conclude, we discuss the results concerning the energy consumption during the switching phases. This energy derives from the charge/discharge of the effective capacitor and from the damped precession of the magnetization [27, 28]. The first contribution $\Delta E_{e}$ represents the so-called $C V^{2}$ dissipation and it can be simply determined when the geometry of the system is given. For example, if we consider the parameters shown in table 1 we obtain $\Delta E_{e}=7.5 \times 10^{-17} \mathrm{~J}$. The second contribution $\Delta E_{m}$ can be evaluated by determining the variation of $\tilde{w}(t)$ during the transitions phases. We can obtain the time evolution of $d \tilde{w}(t) / d t$ through the expression $d \tilde{w}(t) / d t=$ $(\partial \tilde{w} / \partial \vartheta) \dot{\vartheta}+(\partial \tilde{w} / \partial \varphi) \dot{\varphi}$ and we can use Eq.(11) to evaluate the terms $\dot{\vartheta}$ and $\dot{\varphi}$. This procedure can be numerically implemented within the integration scheme of the Langevin system. As before, the average values are determined with the Monte Carlo technique. Since $\tilde{w}$ is always a decreasing function during the switching phases, in Fig.7 (first and second panel) we show the average value of $-d \tilde{w}(t) / d t$ for the transitions B-A and A-B. This is done for 100 equispaced values of $k_{B} T / v$ (from $10 \mathrm{~J} / \mathrm{m}^{3}$ to $4000 \mathrm{~J} / \mathrm{m}^{3}$ ). Interestingly enough, we note that the temperature effects are stronger in the transition A-B. However, we can determine the specific energy dissipated during a transition phase by integrating the time derivative of $\tilde{w}: \Delta E_{m} / v=-\int_{0}^{+\infty}(d \tilde{w}(t) / d t) d t$. The numerical integration leads to the results shown in the third panel of Fig.7, where $\Delta E_{m} / v$ is plotted versus $k_{B} T / v$ for both transitions B-A and A-B. We observe that there is only a very slight (linear) dependence of $\Delta E_{m} / v$ on $k_{B} T / v$. In fact, we can approximate $\Delta E_{m} / v \simeq 2 \times 10^{5} \mathrm{~J} / \mathrm{m}^{3}$ for any value of $k_{B} T / v$. For our original particle with $v=10^{-22} \mathrm{~m}^{3}$ we obtain $\Delta E_{m}=2 \times 10^{-17} \mathrm{~J}$ and the total switching energy is therefore $\Delta E=\Delta E_{e}+\Delta E_{m} \simeq 9.5 \times 10^{-17} \mathrm{~J}$. This value is strongly competitive when 
compared with most non-volatile memory technologies $[15,73,74]$.

\section{Conclusions}

In this work we have developed a complete analysis concerning the effects of the temperature on the magnetization dynamics in a paradigmatic memory element. We considered a heterostructure composed of a magnetoelastic particle embedded in a piezoelectric matrix. In order to introduce the statistical mechanics we have taken into consideration the Langevin equation describing the dynamics of the magnetization vector when the system is in contact with a thermal bath. Its numerical solution allowed us to examine the switching behavior between the stable states in terms of the temperature. We elaborated a blended nanomechanical/magnetic/statistic approach to obtain a comprehensive model and, therefore, a complete picture of its dynamics at finite temperature. The mechanical coupling between magnetoelastic particle and piezoelectric matrix is described by the multi-physics Eshelby formalism, the time evolution of the magnetization orientation is modelled through the Landau-LifshitzGilbert equation and, finally, the presence of the temperature is introduced following the Brown assumption, leading to specific Langevin and Fokker-Planck equations. The thorough combination of these three methods allowed to analyse the most important quantities of the system in terms of the ratio $k_{B} T / v$. More specifically, we determined the switching time, the error probability, and the energy dissipation corresponding to the commutation phases. To conclude, we remark that from the technological point of view the knowledge of these parameters versus $k_{B} T / v$ is crucial for obtaining the desired compromise between maximum operating temperature and size of the memory element (which should be strongly reduced for having a very large-scale integration).

\section{Acknowledgments}

This work is supported by the Agence Nationale de la Recherche ANR (France) through the PNano NAMAMIS project and by the Russian Federation Ministry of Education and Sciences (Contract N. 11.519.11.3023). The authors would like to thank Alain Cappy for fruitful discussions.

\section{Appendix A. Mathematical form of the energy function}

We consider Eqs.(1), (2) and (3) in order to prove Eq.(4). It is important to remark that the local magnetic field $\vec{H}$ and the local stress tensor $\hat{T}$ enter the energy function $w(\vec{\gamma})$ as parameters. It means that the minimization $\min _{\vec{\gamma}:\|\vec{\gamma}\|=1} w(\vec{\gamma} ; \vec{H}, \hat{T})$ furnishes the direction $\vec{\gamma}$ in terms of the magnetic field and the stress tensor, i.e. $\vec{\gamma}=\vec{\gamma}(\vec{H}, \hat{T})$. Therefore, to approach the minimization problem defined by Eq.(1), we can apply the Lagrange method based on the auxiliary function $\mathcal{L}(\vec{\gamma}, \lambda)=w(\vec{\gamma})-\lambda(\vec{\gamma} \cdot \vec{\gamma}-1)$, where $\lambda$ 
is the so-called Lagrange multiplier. Hence, we consider the equations $\partial \mathcal{L} / \partial \gamma_{i}=0$ (for $i=1,2,3)$ and $\partial \mathcal{L} / \partial \lambda=0$. Summing up, we obtain the system

$$
\begin{aligned}
2 \lambda \gamma_{i} & =-\mu_{0} M_{s} H_{i}+\frac{\partial \varphi_{a}(\vec{\gamma})}{\partial \gamma_{i}}-\hat{T}: \frac{\partial \hat{\varepsilon}_{\mu}(\vec{\gamma})}{\partial \gamma_{i}} \\
\vec{\gamma} \cdot \vec{\gamma} & =1 \\
\vec{H} & =\hat{A} \vec{H}^{\infty}+\hat{N} \vec{\gamma} \\
\hat{T} & =\hat{C} \hat{\varepsilon}^{\infty}+\hat{D} \vec{E}^{\infty}+\hat{F}\left[\hat{\varepsilon}_{\mu}(\vec{\gamma})-\hat{\varepsilon}_{\mu}\left(\vec{\gamma}_{0}\right)\right] .
\end{aligned}
$$

We can now substitute the last two relations in the first one, eventually obtaining

$$
\begin{aligned}
& 2 \lambda \gamma_{i}=-\mu_{0} M_{s}\left(\hat{A} \vec{H}^{\infty}+\hat{N} \vec{\gamma}\right)_{i}+\frac{\partial \varphi_{a}(\vec{\gamma})}{\partial \gamma_{i}} \\
& -\left\{\hat{C} \hat{\varepsilon}^{\infty}+\hat{D} \vec{E}^{\infty}+\hat{F}\left[\hat{\varepsilon}_{\mu}(\vec{\gamma})-\hat{\varepsilon}_{\mu}\left(\vec{\gamma}_{0}\right)\right]\right\}: \frac{\partial \hat{\varepsilon}_{\mu}(\vec{\gamma})}{\partial \gamma_{i}}
\end{aligned}
$$

By exploiting the symmetries of tensors $\hat{N}$ and $\hat{F}$ [15] we can simply rewrite Eq.(A.5) as follows (by converting each term in a partial derivative with respect to $\gamma_{i}$ )

$$
\begin{aligned}
& 2 \lambda \gamma_{i}=-\mu_{0} M_{s} \frac{\partial}{\partial \gamma_{i}}\left(\vec{\gamma} \cdot \hat{A} \vec{H}^{\infty}\right)-\frac{1}{2} \mu_{0} M_{s} \frac{\partial}{\partial \gamma_{i}}(\vec{\gamma} \cdot \hat{N} \vec{\gamma}) \\
& +\frac{\partial \varphi_{a}(\vec{\gamma})}{\partial \gamma_{i}}-\frac{\partial}{\partial \gamma_{i}}\left[\hat{C} \hat{\varepsilon}^{\infty}: \hat{\varepsilon}_{\mu}(\vec{\gamma})\right]-\frac{\partial}{\partial \gamma_{i}}\left[\hat{D} \vec{E}^{\infty}: \hat{\varepsilon}_{\mu}(\vec{\gamma})\right] \\
& -\frac{1}{2} \frac{\partial}{\partial \gamma_{i}}\left[\hat{F} \hat{\varepsilon}_{\mu}(\vec{\gamma}): \hat{\varepsilon}_{\mu}(\vec{\gamma})\right]+\frac{\partial}{\partial \gamma_{i}}\left[\hat{F} \hat{\varepsilon}_{\mu}\left(\vec{\gamma}_{0}\right): \hat{\varepsilon}_{\mu}(\vec{\gamma})\right]
\end{aligned}
$$

The previous expression combined with the condition $\vec{\gamma} \cdot \vec{\gamma}=1$ corresponds to a constrained minimization of a new energy function $\tilde{w}$ defined in Eq.(4). We can note that in Eq.(A.6) or (4), while the first term represent the Zeeman contribution (related to the applied magnetic field), the second term represents the self-magnetization of the particle. The factor $1 / 2$ in the second term has been obtained by observing that $\frac{1}{2} \frac{\partial}{\partial \gamma_{i}}(\vec{\gamma} \cdot \hat{N} \vec{\gamma})=(\hat{N} \vec{\gamma})_{i}$. The same argument is valid for the first term in the third line of Eq.(A.6).

\section{Appendix B. The Fokker-Planck equation}

We take into consideration the following system of stochastic differential equations

$$
\frac{d x_{i}(t)}{d t}=h_{i}(\vec{x}, t)+\sum_{j=1}^{m} g_{i j}(\vec{x}, t) n_{j}(t),
$$

where the stochastic processes $n_{j}(t)$ fulfil the following properties

$$
\left\{\begin{array}{l}
\left\langle n_{i}(t)\right\rangle=0 \forall i, t \\
\left\langle n_{i}\left(t_{1}\right) n_{j}\left(t_{2}\right)\right\rangle=2 \delta_{i j} \delta\left(t_{1}-t_{2}\right) \forall i, j, t_{1}, t_{2} \\
n_{i}(t) \text { Gaussian noises. }
\end{array}\right.
$$

If we take into consideration the Stratonovich interpretation of the stochastic calculus, it is possible to prove that the dynamics of the density probability of the state vector $\vec{x}$ 
is described by the following Fokker-Planck equation [40]

$$
\begin{aligned}
\frac{\partial \rho(\vec{x}, t)}{\partial t}= & -\sum_{i=1}^{n} \frac{\partial}{\partial x_{i}}\left[\mathcal{D}_{i}(\vec{x}, t) \rho(\vec{x}, t)\right] \\
& +\sum_{i=1}^{n} \sum_{j=1}^{n} \frac{\partial^{2}}{\partial x_{i} \partial x_{j}}\left[\mathcal{D}_{i j}(\vec{x}, t) \rho(\vec{x}, t)\right],
\end{aligned}
$$

where $D_{i}$ are the so-called drift coefficients given by

$$
\mathcal{D}_{i}(\vec{x}, t)=h_{i}(\vec{x}, t)+\sum_{k=1}^{n} \sum_{j=1}^{m} g_{k j}(\vec{x}, t) \frac{\partial g_{i j}(\vec{x}, t)}{\partial x_{k}} .
$$

Here, the first term represents the standard drift coefficient introduced by the differential problem stated in Eq.(B.1); the second term represents the so-called noise induced drift term and it is a peculiarity of the Stratonovich calculus. We remark that it is different from zero only with multiplicative noises, i.e. when $g_{i j}(\vec{x}, t)$ depends directly on the state $\vec{x}$. On the other hand, the diffusion coefficients $D_{i j}$ are defined as follows

$$
\mathcal{D}_{i j}(\vec{x}, t)=\sum_{k=1}^{m} g_{i k}(\vec{x}, t) g_{j k}(\vec{x}, t) .
$$

They take into account the fluctuations introduced by the noise terms. Through this theory, in Section 3 we have established the Fokker-Planck equation associated to the Langevin systems given in Eqs.(6) and (11) and we have found the same evolution equation given in Eq.(7). We remark that, while for the Langevin system in Eq.(6) we obtain a noise induced drift term represented by the third line in Eq.(7), for the simplified version of the Langevin system in Eq.(11) the noise induced drift term is zero, leading to the equivalence of the Itô and the Stratonovich interpretation for this particular case.

\section{Appendix C. On stochastic differential equations}

The stochastic differential equation defined in Eq.(B.1) can be integrated in a time step, by obtaining

$$
\begin{aligned}
x_{i}(t+\delta t)-x_{i}(t) & =\int_{t}^{t+\delta t} h_{i}(\vec{x}, t) d t \\
& +\int_{t}^{t+\delta t} g_{i j}(\vec{x}, t) n_{j}(t) d t,
\end{aligned}
$$

where the sum over $j$ is implicit for brevity and the noises satisfy properties in Eq.(B.2). We adopt a general integration rule that takes into account both the Itô $(\alpha=0)$ and the Stratonovich $(\alpha=1 / 2)$ approaches (also including all the intermediate cases): in the integrands we substitute $\vec{x} \rightarrow(1-\alpha) \vec{x}(t)+\alpha \vec{x}(t+\delta t)$, i.e. a weighted means of the values assumed at the endpoints of the interval. We can define $\delta \vec{x}=\vec{x}(t+\delta t)-\vec{x}(t)$ and, therefore, we can rewrite the substitution as $\vec{x} \rightarrow \vec{x}(t)+\alpha \delta \vec{x}$. For the first integral 
in Eq.(C.1) we obtain

$$
\int_{t}^{t+\delta t} h_{i}(\vec{x}, t) d t \cong h_{i}(\vec{x}(t), t) \delta t+\frac{\partial h_{i}}{\partial x_{k}}(\vec{x}(t), t) \alpha \delta x_{k} \delta t
$$

and, similarly, for the second one we get

$$
\begin{aligned}
& \int_{t}^{t+\delta t} g_{i j}(\vec{x}, t) n_{j}(t) d t \cong\left[g_{i j}(\vec{x}(t), t)\right. \\
& \left.+\frac{\partial g_{i j}}{\partial x_{k}}(\vec{x}(t), t) \alpha \delta x_{k}\right] \int_{t}^{t+\delta t} n_{j}(t) d t .
\end{aligned}
$$

Summing up, we have found the first form of the integration scheme

$$
\begin{aligned}
\delta x_{i}= & h_{i}(\vec{x}(t), t) \delta t+\frac{\partial h_{i}}{\partial x_{k}}(\vec{x}(t), t) \alpha \delta x_{k} \delta t \\
& +g_{i j}(\vec{x}(t), t) \int_{t}^{t+\delta t} n_{j}(t) d t \\
& +\frac{\partial g_{i j}}{\partial x_{k}}(\vec{x}(t), t) \alpha \delta x_{k} \int_{t}^{t+\delta t} n_{j}(t) d t .
\end{aligned}
$$

The term $\int_{t}^{t+\delta t} n_{j}(t) d t$ is a Gaussian random variable with the following expectation values

$$
\begin{aligned}
& \left\langle\int_{t}^{t+\delta t} n_{j}(t) d t\right\rangle=0 \\
& \left\langle\int_{t}^{t+\delta t} n_{i}(t) d t \int_{t}^{t+\delta t} n_{j}(\tau) d \tau\right\rangle \\
& =\int_{t}^{t+\delta t} \int_{t}^{t+\delta t}\left\langle n_{i}(t) n_{j}(\tau)\right\rangle d t d \tau=2 \delta_{i j} \delta t .
\end{aligned}
$$

So, we can define

$$
P_{j}=\frac{1}{\sqrt{2 \delta t}} \int_{t}^{t+\delta t} n_{j}(t) d t,
$$

and we obtain a sequence of Gaussian random variables with the properties $\left\langle P_{j}\right\rangle=0$ and $\left\langle P_{i} P_{j}\right\rangle=\delta_{i j}$. To complete the calculation we have to re-substitute Eq.(C.4) into itself and to retain only the terms of order $\delta t$. When we make the substitution in the first term containing $\delta x_{k}$ (first line in Eq.(C.4)), we observe that the term itself disappears because all coefficients are of order larger than $\delta t$. Differently, when we make the substitution in the second term containing $\delta x_{k}$ (third line in Eq.(C.4)), we observe that only one term must be considered. More specifically, we must retain the term with the product between $\int_{t}^{t+\delta t} n_{s}(t) d t$ and $\int_{t}^{t+\delta t} n_{j}(t) d t$; in fact, both term are proportional to $\sqrt{\delta t}$, generating a product of order $t$, which is not negligible. Explicitly, we obtain

$$
\begin{aligned}
& \delta x_{i}=h_{i}(\vec{x}(t), t) \delta t+g_{i j}(\vec{x}(t), t) \sqrt{2 \delta t} P_{j} \\
& +\frac{\partial g_{i j}}{\partial x_{k}}(\vec{x}(t), t) \alpha g_{k s}(\vec{x}(t), t) \int_{t}^{t+\delta t} n_{s}(t) d t \int_{t}^{t+\delta t} n_{j}(t) d t .
\end{aligned}
$$


Now, the average value of the product of the two integrals is given in Eq.(C.6) and the result is $2 \delta_{s j} \delta t$. Moreover, it is simple to verify that the second order expectation values of the same quantity,

$$
\begin{aligned}
\int_{t}^{t+\delta t} \int_{t}^{t+\delta t} \int_{t}^{t+\delta t} \int_{t}^{t+\delta t} & \left\langle n_{s}\left(t_{1}\right) n_{j}\left(t_{2}\right) n_{q}\left(t_{3}\right) n_{i}\left(t_{4}\right)\right\rangle \\
& \times d t_{1} d t_{2} d t_{3} d t_{4}
\end{aligned}
$$

is equal to zero for: (i) four different indices $s, j, q$ and $i$, (ii) $s=j=q \neq i$, and (iii) $s=j \neq q, s=j \neq i$ and $q \neq i$. Moreover, we have the result $4 \delta t^{2}$ for $s=j \neq q=i$ and the result $12 \delta t^{2}$ for $s=j=q=i$. Of course all results are invariant to any indices permutation. So, the second order expectation values are zero or of order $\delta t^{2}$. Therefore, we may assume the average value as the deterministic value of the product. The final result corresponds to the Euler scheme of integration for a stochastic differential equation

$$
\begin{aligned}
\delta x_{i}= & {\left[h_{i}(\vec{x}(t), t)+2 \alpha \frac{\partial g_{i j}}{\partial x_{k}}(\vec{x}(t), t) g_{k j}(\vec{x}(t), t)\right] \delta t } \\
& +g_{i j}(\vec{x}(t), t) \sqrt{2 \delta t} P_{j} .
\end{aligned}
$$

In the specific case of the Stratonovich interpretation we have $\alpha=1 / 2$ and the integration scheme becomes

$$
\delta x_{i}=D_{i}(\vec{x}(t), t) \delta t+g_{i j}(\vec{x}(t), t) \sqrt{2 \delta t} P_{j},
$$

where the drift coefficients $D_{i}$, defined in Eq.(B.4), are perfectly coherent with the Fokker-Planck equation. In order to implement the integration scheme of Eq.(11) we need two Gaussian random variables at any time step and, therefore, they can be generated through the Box-Muller theorem: if $U_{1}$ and $U_{2}$ are independent random variables uniformly distributed in $(0,1)$, then $P_{1}=\left(-2 \log U_{1}\right)^{1 / 2} \cos \left(2 \pi U_{2}\right)$ and $P_{2}=$ $\left(-2 \log U_{1}\right)^{1 / 2} \sin \left(2 \pi U_{2}\right)$ are Gaussian independent random variables with $\left\langle P_{j}\right\rangle=0$ and $\left\langle P_{i} P_{j}\right\rangle=\delta_{i j}$, as requested in Eq.(C.11). Finally, we underline that the convergence of the Euler scheme to the solution of Eq.(B.1) is assured by the Skorokhod theorem $[57,75]$.

\section{References}

[1] Fiebig M 2005 Revival of the magnetoelectric effect J. Phys. D: Appl. Phys. 38123

[2] Eerenstein W et al 2006 Multiferroic and magnetoelectric materials Nature 442759

[3] Ramesh R and Spaldin N A 2007 Multiferroics: progress and prospects in thin films Nat. Mater. 621

[4] Garcia V et al 2010 Ferroelectric Control of Spin Polarization Science 327106

[5] Lawes G and Srinivasan G 2011 Introduction to magnetoelectric coupling and multiferroic films $J$. Phys. D: Appl. Phys. 44243001

[6] Nan C W et al 2008 Multiferroic magnetoelectric composites: Historical perspective, status, and future directions J. Appl. Phys. 103031101

[7] Wang Y et al 2010 Multiferroic magnetoelectric composite nanostructures NPG Asia Mater. 261

[8] D'Souza N et al 2011 Four-state nanomagnetic logic using multiferroics J. Phys. D: Appl. Phys. 44265001 
[9] Pertsev N A and Kohlstedt H 2010 Resistive switching via the converse magnetoelectric effect in ferromagnetic multilayers on ferroelectric substrates Nanotechnology 21475202

[10] Roy K et al 2011 Hybrid spintronics and straintronics: A magnetic technology for ultra low energy computing and signal processing Appl. Phys. Lett. 99063108

[11] Khvalkovskiy A V et al 2013 Basic principles of STT-MRAM cell operation in memory arrays $J$. Phys. D: Appl. Phys. 46074001

[12] Chiabrera A et al 1989 Physical limits of integration and information processing in molecular systems J. Phys. D: Appl. Phys. 221571

[13] NamKoong J H and Lim S H 2009 Temperature increase in nanostructured cells of a magnetic tunnel junction during current-induced magnetization switching J. Phys. D: Appl. Phys. 42 225003

[14] Tiercelin N et al 2011 Magnetoelectric memory using orthogonal magnetization states and magnetoelastic switching J. Appl. Phys. 109 07D726

[15] Giordano S et al 2012 Combined nanomechanical and nanomagnetic analysis of magnetoelectric memories Phys. Rev. B 85155321

[16] Tiercelin N et al 2011 Room temperature magnetoelectric memory cell using stress-mediated magnetoelastic switching in nanostructured multilayers Appl. Phys. Lett. 99192507

[17] Dusch Y et al 2013 Stress-mediated magnetoelectric memory effect with uniaxial $\mathrm{TbCo}_{2} / \mathrm{FeCo}$ multilayer on 011-cut PMN-PT ferroelectric relaxor J. Appl. Phys. 113 17C719

[18] Tiercelin N et al Mémoire magnétoélectrique (MELRAM), Patent FR2961632 (A1), submitted 18-06-2010, published 23-12-2011. Magnetoelectric Memory, Patent Extension PCT WO/2011/158208, submitted 17-06-2011, published 22-12-2011.

[19] Chu Y H et al 2008 Electric-field control of local ferromagnetism using a magnetoelectric multiferroic Nat. Mater. 7478

[20] He X et al 2010 Robust isothermal electric control of exchange bias at room temperature Nat. Mater. 9579

[21] Heron J T et al 2011 Electric-Field-Induced Magnetization Reversal in a Ferromagnet-Multiferroic Heterostructure Phys. Rev. Lett. 107217202

[22] Chiba D et al 2008 Magnetization vector manipulation by electric fields Nature 455515

[23] Chiba D et al 2010 Simulation of magnetization switching by electric-field manipulation of magnetic anisotropy Appl. Phys. Lett. 96, 192506

[24] Stöhr J et al 2009 Magnetization switching without charge or spin currents Appl. Phys. Lett. 94 072504

[25] Maruyama T et al 2009 Large voltage-induced magnetic anisotropy change in a few atomic layers of iron Nat. Nano 4158

[26] Balestriere P et al 2010 Electric field induced anisotropy modification in (Ga,Mn)As: A strategy for the precessional switching of the magnetization Appl. Phys. Lett. 96142504

[27] Roy K et al 2011 Switching dynamics of a magnetostrictive single-domain nanomagnet subjected to stress Phys. Rev. B $\mathbf{8 3} 224412$

[28] Roy K et al 2012 Energy dissipation and switching delay in stress-induced switching of multiferroic nanomagnets in the presence of thermal fluctuations J. Appl. Phys. 112023914

[29] Asaro R J and Lubarda V A 2006 Mechanics of Solids and Materials (Cambridge: Cambridge University Press)

[30] Colombo L and Giordano S 2011 Nonlinear elasticity in nanostructured materials Rep. Progr. Phys. 74116501

[31] Eshelby J D 1957 The Determination of the Elastic Field of an Ellipsoidal Inclusion, and Related Problems Proc. R. Soc. London A241 376

[32] Huang J H and Kuo W S 1997 The analysis of piezoelectric/piezomagnetic composite materials containing ellipsoidal inclusions J. Appl. Phys. 811378

[33] Landau L and Lifshitz E 1935 On the Theory of the Dispersion of Magnetic Permeability in Ferromagnetic Bodies Phys. Z. Sowjetunion 8153 
[34] Gilbert T L 1955 A Lagrangian formulation of the gyromagnetic equation of the magnetic field Phys. Rev. 1001243 (abstract only); Gilbert T L 2004 A phenomenological theory of damping in ferromagnetic materials IEEE Trans. Mag. 403443

[35] Brown W F 1959 Relaxational Behavior of Fine Magnetic Particles J. Appl. Phys. 30 S130

[36] Brown W F 1963 Thermal Fluctuations of a Single-Domain Particle J. Appl. Phys. 341319

[37] Brown W F 1963 Thermal Fluctuations of a Single-Domain Particle Phys. Rev. 1301677

[38] Brown W F 1979 Thermal fluctuation of fine ferromagnetic particles IEEE Trans. Magn. 151196

[39] Hänggi P and Thomas H 1982 Stochastic Processes: Time-Evolution, Symmetries and Linear Response Phys. Rep. 88207

[40] Risken H 1989 The Fokker-Planck equation (Berlin: Springer Verlag)

[41] Coffey W T et al 2004 The Langevin equation (Singapore: World Scientific)

[42] Daniel L et al 2008 Reversible magneto-elastic behavior: a multiscale approach J. Mech. Phys. Sol. 561018

[43] West F G 1964 Uniaxial Anisotropy due to Magnetoelastic Energy in Constrained Polycrystalline Films J. Appl. Phys. 351827

[44] Giordano S and Palla P L 2008 Dielectric behavior of anisotropic inhomogeneities: interior and exterior points Eshelby tensors J. Phys. A: Math. Theor. 41415205

[45] Giordano S 2003 Effective medium theory for dispersions of dielectric ellipsoids J. Electrost. 5859

[46] Heinonen E et al 2005 Characterization and modelling of 3D piezoelectric ceramic structures with ATILA software J. Eur. Ceram. Soc. 252467

[47] Huang J H et al 1998 Magneto-electro-elastic Eshelby tensors for a piezoelectric-piezomagnetic composite reinforced by ellipsoidal inclusions J. Appl. Phys. 835364

[48] Mikata Y 2001 Explicit determination of piezoelectric Eshelby tensors for a spheroidal inclusion Int. J. Sol. Struct. 387045

[49] Li J Y and Dunn M L 1998 Anisotropic coupled-field inclusion and inhomogeneity problems Phil. Mag. A 771341

[50] Huang Y Y and Jin Y M 2008 Phase field modeling of magnetization processes in growth twinned Terfenol-D crystals Appl. Phys. Lett. 93142504

[51] Lakshmanan M 2011 The fascinating world of the LandauLifshitzGilbert equation: an overview Phil. Trans. R. Soc. A 3691280

[52] Uhlenbeck G E and Ornstein L S 1930 On the Theory of the Brownian Motion Phys. Rev. 36823

[53] Wang M C and Uhlenbeck G E 1945 On the Theory of the Brownian Motion II Rev. Mod. Phys. 17323

[54] Caldirola P and Lugiato L A 1982 Connection between the Schrödinger equation for dissipative systems and the master equation Physica A 116248

[55] Bianco B et al 2001 Friction and noise in quantum mechanics: a model for the interactions between a system and a thermal bath Il Nuovo Cimento 116155

[56] Schuss Z 1980 Theory and Applications of Stochastic Differential Equations (New York: John Wiley \& Sons)

[57] Schuss Z 2010 Theory and Applications of Stochastic Processes (New York: Springer)

[58] Denisov S I et al 2007 Rapidly driven nanoparticles: Mean first-passage times and relaxation of the magnetic moment Phys. Rev. B 75184432

[59] Bertotti G et al 2000 Nonlinear Magnetization Dynamic in Nanosystems (Oxford:Elsevier)

[60] Fredkin D R 2001 Brownian motion on manifolds, with application to thermal magnetization reversal Physica B 30626

[61] Box G E P and Muller M E 1958 A Note on the Generation of Random Normal Deviates Ann. Math. Stat. 29610

[62] Fukushima $\mathrm{H}$ et al 2002 Numerical solutions of the FokkerPlanck equation by the finite difference method for the thermally assisted reversal of the magnetization in a single-domain particle $J$. Magn. Mag. Mat. 2421002

[63] Fukushima $\mathrm{H}$ et al 2002 Dependence of switching time on temperature, applied field, and material 
parameters IEEE Trans. Magn. 382394

[64] Fukushima $\mathrm{H}$ et al 2004 Effects of damping constant and gyromagnetic term on switching time of single-domain particle J. Magn. Mag. Mat. 272745

[65] Kalmykov Yu P 1996 Rotational Brownian Motion in an External Potential: The Langevin Equation Approach J. Mol. Liq. 69117

[66] Coffey W T and Geoghegan L J 1996 Differential recurrence relations for the Fokker-Planck equation for Magnetic after-effect and dielectric relaxation in non axially symmetric potentials J. Mol. Liq. 6953

[67] Kalmykov Yu P and Titov S V 1999 Matrix elements of the system of moment equations governing the kinetics of superparamagnetic particles Phys. Rev. Lett. 822967

[68] Coffey W T et al 2000 Interpolation formulae between axially symmetric and non-axially symmetric Kramers' escape rates for single-domain ferromagnetic particles in the intermediate to highdamping limit J. Magn. Mag. Mat. 221110

[69] Déjardin P M et al 2001 Interpolation formula between very low and intermediate-to-high damping Kramers escape rates for single-domain ferromagnetic particles Phys. Rev. E 63021102

[70] Kalmykov Yu P et al 2005 Damping dependence of the magnetization relaxation time of singledomain ferromagnetic particles J. Magn. Mag. Mat. 292372

[71] Ouari B and Kalmykov Yu P 2006 Dynamics of the magnetization of single domain particles having triaxial anisotropy subjected to a uniform dc magnetic field J. Appl. Phys. 100, 123912

[72] García-Palacios J L and Lázaro F J 1998 Langevin-dynamics study of the dynamical properties of small magnetic particles Phys. Rev. B 5814937

[73] Wang K L et al 2013 Low-power non-volatile spintronic memory: STT-RAM and beyond J. Phys. D: Appl. Phys. 46074003

[74] Kryder M H and Kim C S 2009 After Hard Drives - What Comes Next? IEEE Trans. Magn. 45 3406

[75] Skorokhod A V 1961 Stochastic Equations for Diffusion Processes in a Bounded Region Theor. Probab. Appl. 6264 